\title{
Cimentos álcali ativados: efeito da cura térmica no desenvolvimento da resistência mecânica
}

\author{
Activated alkali cements: effect of thermal \\ curing on the development of mechanical \\ strength
}

Andreza Frare ${ }^{1}$, Caroline Angulski da Luz ${ }^{2}$

\author{
${ }^{1}$ Programa de Pós-graduação em Engenharia Civil - PPGECC/UTFPR, CEP: 571- 85503- 390, Pato Branco, PR, Brasil. \\ e-mail: frareandreza@gmail.com \\ ${ }^{2}$ Programa de Pós-graduação em Engenharia Civil - PPGECC/UTFPR, CEP: 571- 85503- 390, Pato Branco, PR, Brasil. \\ e-mail: angulski@utfpr.edu.br
}

\section{RESUMO}

Devido à extração de matérias primas não renováveis e ao lançamento de $\mathrm{CO} 2$ na atmosfera pela produção de Cimento Portland, os cimentos álcali-ativados (CAT) vêm ganhando espaço no cenário mundial já que o seu processo de obtenção não envolve calcinação dos matérias-primas e utiliza diversos subprodutos. Assim, eles são considerados como um produto de baixo impacto ambiental e com boas propriedades de engenharia. Os CAT's podem ser obtidos majoritariamente de escória de alto forno, a qual pode ter de origem em fornos a carvão vegetal ou a coque, o que modifica sua composição química. O objetivo deste trabalho foi avaliar a influência da composição química da escória de alto no comportamento do CAT e investigar a eficiência da cura térmica no melhoramento da resistência à compressão. Para a produção do CAT, foram empregadas duas escórias, A e B, provenientes de fornos a carvão vegetal e a coque, respectivamente, além do hidróxido de sódio como ativador alcalino. As amostras foram submetidas à cura térmica por um período de $24 \mathrm{~h}$ nas temperaturas de $400 \mathrm{C}$ e $600 \mathrm{C}$ e avaliadas nos testes de resistência à compressão, de calorimetria e de análises de microestrutura aos 3, 7 e 28 dias. Os resultados mostraram que a escória A se mostrou mais reativa e mais influenciada pela temperatura de cura. As argamassas feitas com escória A (CAT/A) apresentaram desempenho mecânico superior ao da escória B (CAT/B), em todas as temperaturas. As análises de microestrutura mostraram que no CAT/A houve formação majoritária de CSH, enquanto que no CAT/B, de CSH e de hidrotalcita. A cura térmica não foi eficiente para melhorar o comportamento mecânico da do CAT/B.

Palavras-chave: cimento álcali ativado, escória de alto forno, cura térmica.

\begin{abstract}
Due to the extraction of nonrenewable raw materials and the release of $\mathrm{CO} 2$ into the atmosphere by the production of Portland cement, the alkali-activated cements (AAC) have been gaining ground in the world scenario since their production process does not involve calcination of the raw materials and uses various byproducts. Thus, they are considered as a low environmental impact product and with good engineering properties. The AAC's can be obtained mainly from blast furnace slag (BFS), which may have origin in charcoal ovens or coke, which modifies their chemical composition. The objective of this work was to evaluate the influence of the chemical composition of BFS on the behavior of the AAC and to investigate the thermal cure efficiency in the improvement of the compressive strength. For the production of AAC, two slag, A and B, were used from charcoal kilns and coke, respectively, in addition to sodium hydroxide as an alkaline activator. The samples were subjected to thermal cure for a period of 24 hours at temperatures of $40 \mathrm{oC}$ and $60 \mathrm{o} \mathrm{C}$, which were subjected to the compressive strength, calorimetry and microstructure analyzes at 3, 7 and 28 days. The results showed that slag A was more reactive and more influenced by the cure temperature. The AAC made with BFS- A (AAC / A) slag presented higher mechanical performance than B (AAC / B) at at all temperatures. Microstructure analyzes showed that in AAC/A there was a majority formation of CSH, while
\end{abstract}


in CAT/B, CSH and hydrotalcite. Thermal curing was not efficient to improve the mechanical behavior of $\mathrm{CAT} / \mathrm{B}$.

Keywords: alkali activated cement, blast furnace slag, thermal curing.

\section{INTRODUÇÃO}

O cimento Portland é um dos materiais mais utilizados pela construção civil, porém, a extração de matérias primas não renováveis, o elevado consumo de energia decorrente da calcinação do clínquer e as taxas de $\mathrm{CO}_{2}$ liberadas em sua produção tem se tornado um aspecto negativo do ponto de vista econômico e ambiental $[1,2]$.

Estima -se que para produção de uma tonelada de clínquer despeja-se uma tonelada de $\mathrm{CO}_{2}$ na atmosfera, e extrai-se 1,15 toneladas de calcário das reservas naturais [3]. Pensando-se na redução dessas emissões e no aumento da disponibilidade de matéria - prima, estudos direcionados a cimentos baixo impacto ambiental têm ganhado força no cenário mundial, como é o caso do cimento álcali-ativado (CAT).

Os cimentos álcali-ativados (CAT's) apresentam características como alta resistência, durabilidade e baixo impacto ambiental e são compostos basicamente por subprodutos ou resíduos industriais, tais como cinza volante e escória de alto forno. Estes materiais são compostos basicamente por alumina $\left(\mathrm{Al}_{2} \mathrm{O}_{3}\right)$, sílica $\left(\mathrm{SiO}_{2}\right)$, óxido de cálcio $(\mathrm{CaO})$, os quais são ativados por uma solução alcalina, formando como produto principal o gel CSH (JUENGER, WINNEFELD, et al., 2011) [4].

A escória de alto forno pode ser proveniente de fornos a carvão vegetal ou a coque, sendo a primeira economicamente mais viável por utilizar uma fonte de energia renovável e independe das importações. A duas diferem em sua composição química e na relação entre seus componentes principais [5, 6].

As escórias podem ainda ser classificadas em ácidas e básicas dependendo da relação $\mathrm{CaO}-\mathrm{SiO}_{2}$. Geralmente a escória a carvão vegetal é considerada ácida por conter sílica como componente predominante, e portanto, sua relação $\left(\mathrm{CaO}-\mathrm{SiO}_{2}\right)$ é menor que um; enquanto a escória a coque é considerada básica por apresentar a mesma relação maior que um $[5,6,7]$.

A reatividade da escória depende em geral de suas propriedades, como composição química, forma de resfriamento, finura e também do tipo de ativador alcalino em geral hidróxido de sódio $(\mathrm{NaOH})$ ou silicato de sódio $\left(\mathrm{Na}_{2} \mathrm{SiO}_{3}\right)[8,9]$. Estudos avaliando diferentes composições químicas das escórias na produção de CAT, constataram que maiores teores de $\mathrm{MgO}$ em sua composição resultam em maiores resistências à compressão e formação de hidrotalcita. Já matérias - primas com maior concentração de $\mathrm{Al}_{2} \mathrm{O}_{3}$ apresentaram menor calor de hidratação, sem influência na resistência, além de apresentar maiores teores de Al no gel C-S-H [10, $11,12,13]$. Num trabalho recente, Langaro et al [2] verificaram que CAT formulado com escória ácida, gerada em forno a carvão vegetal, apresentaram resistência mecânica superior a $40 \mathrm{MPa}$, enquanto que a básica, gerada em forno a coque, mostrou valores máximos de $10 \mathrm{MPa}$. A escória ácida, com maior teor de $\mathrm{MgO}$, também apresentou maior resistência mecânica, mas a hidrotalcita foi observada apenas na escória básica. $\mathrm{O}$ efeito da finura da escória também foi observado e os autores não notaram influência significativa do aumento da superfície específica da escória ácida, de 3300 para $5300 \mathrm{~cm}^{2} / \mathrm{g}$, na resistência à compressão.

As propriedades dos cimentos álcali-ativados e de outros cimentos de escória, são também afetadas pelos efeitos da temperatura. O calor é geralmente aplicado como meio de acelerar as reações químicas melhorando o desempenho mecânico, tanto em cimentos convencionais quanto em CAT's [14, 15].

As temperaturas de cura também afetam os geopolímeros, classificados como um subgrupo dos CAT's que utilizam em sua composição materiais como cinzas, metacaulins e argilas, formando sistemas alcalinos, compostos basicamanete por $\mathrm{SiO}_{2}, \mathrm{Al}_{2} \mathrm{O}_{3}$ e apresentam maiores concentrações dos elementos sódio (Na) e potássio (K), desenvolvendo como produto da reação aluminossilicatos hidratado (N-A-S-H). Estudos com variações de temperatura em geopolímeros têm mostrado o aumento do desempenho mecânico quando curados em temperaturas de 40 à $70^{\circ} \mathrm{C}$, durante as primeiras horas de hidratação. Isto se deve à maior formação de gel C-S-H [16, 17, 18, 19, 20]. Além disso, a estrutura dos geopolímeros submetidos às temperaturas mais elevadas apresentou-se mais densa e compacta, enquanto que aqueles curados em temperaturas inferiores à $40^{\circ} \mathrm{C}$ apresentavam-se mais porosos [19]. Porém efeitos adversos também podem ocorrer quando a temperatura de cura excede esses valores, bem como longos períodos de exposição, causando perda de umidade afetando assim o processo de hidratação e formação dos compostos [21, 22].

Esse artigo investigou os micromecanismos e resistência à compressão dos cimentos álcali-ativados, submetidos a três temperaturas de cura, e a relação com a composição química da escória de alto forno. 


\section{MATERIAIS E MÉTODOS}

\subsection{Materiais}

Para formular os cimentos álcali ativados, foram empregadas duas escórias granuladas de alto forno, A e B, proveniente de fornos a carvão vegetal e a coque, respectivamente. As escórias passaram por um processo de secagem à $105{ }^{\circ} \mathrm{C}$ por um período de 24 horas, ambas trituradas por 2 horas em moinho de bolas. Após, foram determinadas a composição química, através de fluorescência de raios X , difração de raios X (DRX), distribuição granulometria a laser, massa específica e superfície específica Blaine (NBR 16372) [23].

Tabela 1: Composição química da Escória A e B (\%).

\begin{tabular}{lll}
\hline COMPONENTE & ESCÓRIA A & ESCÓRIA B \\
\hline $\mathrm{SiO}_{2}$ & 38,1 & 32,2 \\
\hline $\mathrm{Al}_{2} \mathrm{O}_{3}$ & 13,9 & 8,2 \\
\hline $\mathrm{Fe}_{2} \mathrm{O}_{3}$ & 1,3 & 0,8 \\
\hline $\mathrm{CaO}$ & 37,0 & 49,5 \\
\hline $\mathrm{MgO}$ & 6,2 & 5,0 \\
\hline $\mathrm{SO}_{3}$ & 0,1 & 1,4 \\
\hline $\mathrm{Na}_{2} \mathrm{O}$ & 0,2 & 0,1 \\
\hline $\mathrm{TiO}_{2}$ & 0,8 & 0,6 \\
\hline $\mathrm{MnO}$ & 1,1 & 1,3 \\
\hline $\mathrm{CaO} / \mathrm{SiO}_{2}$ & 0,97 & 1,54 \\
\hline
\end{tabular}

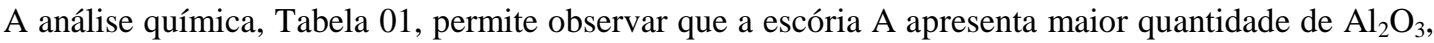
$\mathrm{MgO}$ e $\mathrm{SiO}_{2}$ e menor quantidade de $\mathrm{CaO}$ que a escória $\mathrm{B}$. O maior teor de $\mathrm{CaO}$ é característico das escórias geradas em fornos a coque (A) [7]. Assim, através da relação $\mathrm{CaO} / \mathrm{SiO}_{2}$, a escória A foi classificada com ácida e B como básica, como esperado.

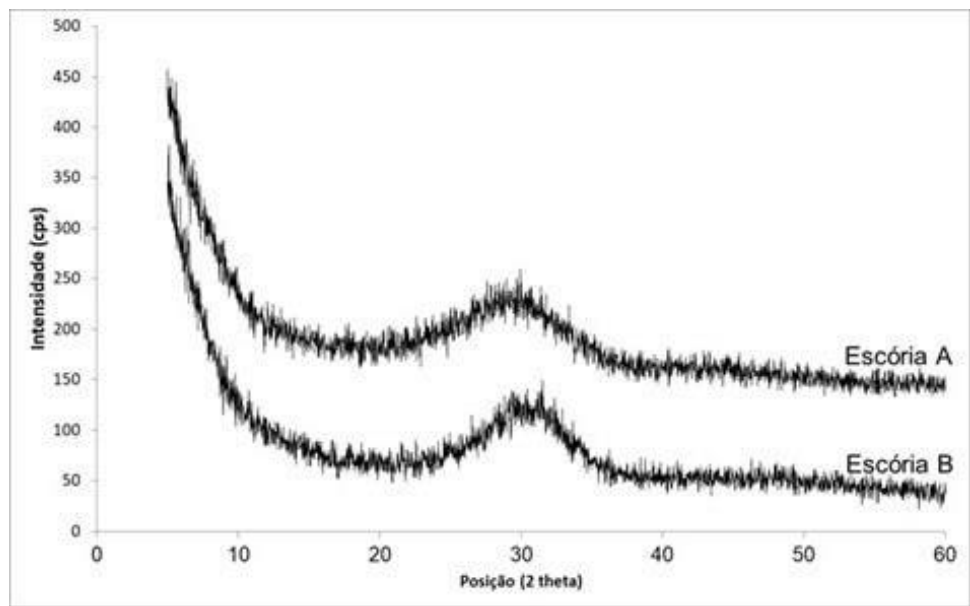

Figura 1: Difração de raio-X escórias A e B.

Os difratogramas da escória A e B, apresentados na Figura 1, são similares e não apresentam picos definidos, indicando um material amorfo, o que contribui para a reatividade do material.

A Tabela 2 mostra que as massas específicas e superfícies específicas das escórias são semelhantes, mas a curva granulométrica, exibida na Figura 2, indica que a escória A é mais fina, com diâmetros d50 e

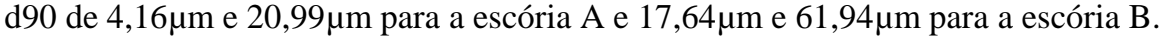


Tabela 2: Caracterização física das escórias A e B.

\begin{tabular}{lll}
\hline ESCÓRIA & MASSA ESPECÍFICA $\left(\mathbf{g} / \mathbf{c m}^{3}\right)$ & SUPERFÍCIE ESPECÍFICA $\left(\mathbf{c m}^{2} / \mathbf{g}\right)$ \\
\hline $\mathbf{A}$ & 2,85 & 4361, \\
\hline B & 2,92 & 4677 \\
\hline
\end{tabular}

Figura 2: Granulometria a laser das escórias A e B.

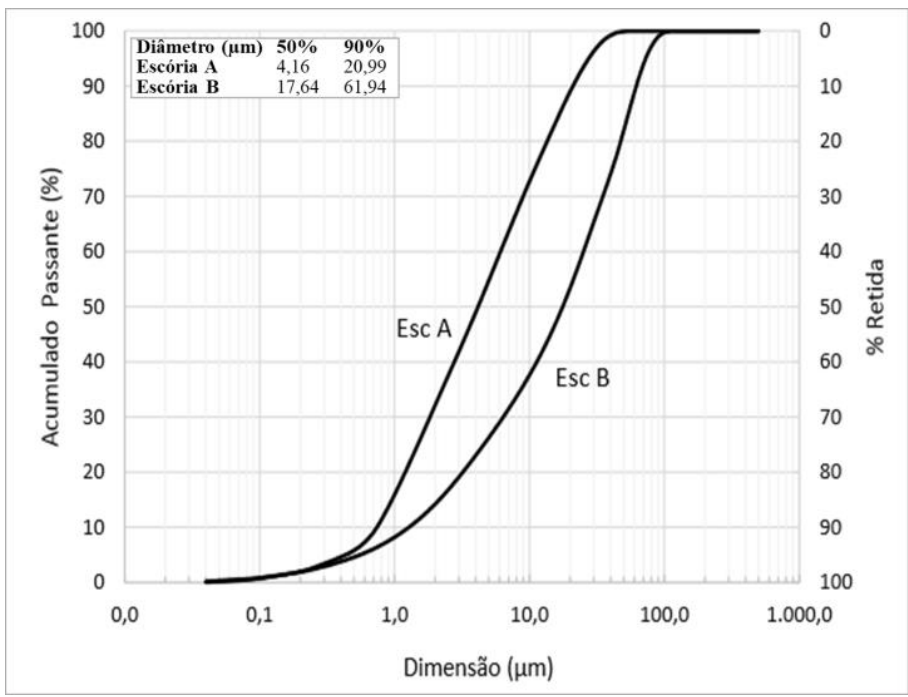

O ativador alcalino utilizado foi o hidróxido de sódio $(\mathrm{NaOH})$, da marca Sodabel e pureza de $99 \%$.

A areia emprega foi a normal, proveniente do Instituto de Pesquisa Tecnológica (IPT), a qual é separada quatro frações granulométricas, ou seja, material retido entre peneiras de abertura nominal de $2,4 \mathrm{~mm}$ e $1,2 \mathrm{~mm}$ (grossa); $1,2 \mathrm{~mm}$ e $0,6 \mathrm{~mm}$ (média grossa); $0,6 \mathrm{~mm}$ e $0,3 \mathrm{~mm}$ (média fina) e 0,3 $\mathrm{mm}$ e $0,15 \mathrm{~mm}$ (fina).

\subsection{Métodos}

Para a obtenção do cimento, utilizou-se o $\mathrm{NaOH}$ na proporção de $5 \%$ em relação à massa de escória, conforme Langaro, et al. [2].

A cura térmica foi baseada no trabalho de Suwan, et al. [19]. O método consistiu em manter os corpos de prova pelo período inicial de 24 horas em cura úmida (95\% umidade) nas temperaturas de 23,40 e $60{ }^{\circ} \mathrm{C}$. Após esse período, as amostras mantiveram-se em cura úmida mas à temperatura de $23{ }^{0} \mathrm{C}$ até as idades de realização dos ensaios.

\subsubsection{Resistência mecânica}

Para avaliar a influência da cura na resistência mecânica do cimento álcali-ativado foram confeccionadas argamassas de 1: 2,75: 0,485 (escória: agregado: água, em massa), de acordo com a ASTM C 348-02 [24]. Os corpos de prova foram submetidos à cura térmica e rompidas com 24 horas, 3,7 e 28 dias.

\subsubsection{Avaliação microestrutural}

Para os ensaios de avaliação microestrutural foram confeccionadas pastas com relação água-escória de 0,4 [2], as quais foram submetidas ao mesmo processo de cura térmica das argamassas e avaliadas aos 7 e 28 dias.

O processo de interrupção da hidratação consistiu fraturá-las e colocá-las imersas em acetona por 2 horas. Posteriormente retirou-se o excesso de acetona através do filtro de buchner acoplado à bomba de vácuo. Para a completa retirada de acetona colocou-se as mostras em estufa por 24 horas à temperatura de $40{ }^{\circ} \mathrm{C}$, as quais foram fragmentas em pedaços menores para o ensaio de Microscopia de Varredura Eletrônica (MEV) e em granulometria inferior a $150 \mu \mathrm{m}$ para calorimetria exploratória diferencial (DSC) e difração de raios-X (DRX). Todo o procedimento foi baseado no trabalho de Langaro et al. [2]. 
As análises de MEV foram realizadas, sob corrente de $15 \mathrm{Kv}$ e aumento de $2000 \mathrm{x}$. Para análise DSC utilizou-se o equipamento da marca TA Instruments, modelo DSC Q20 com faixa de aquecimento de 30 a $600{ }^{\circ} \mathrm{C}$, sob ar sintético com fluxo de $100 \mathrm{~mL} \mathrm{~min}^{-1}$ e porta amostra de alumínio fechado com um furo na tampa. Para a realização do ensaio de DRX utilizou-se o equipamento da marca Panalytical, modelo EMPYREAN, o qual utiliza um tubo de radiação $\mathrm{Cu}-\mathrm{K} \alpha,(40 \mathrm{kV}, 25 \mathrm{~mA})$. As amostras foram submetidas a um comprimento de onda de $1,54 \breve{A}$, ângulos de varredura de $5-70^{\circ}(2 \theta)$ e passo de $0,02^{\circ}$, sendo as intensidades registradas por 10 s em cada passo [2].

\section{RESULTADOS}

A Figura 3 representa os resultados de resistência à compressão para os CAT feitos escórias A e B, curados por $24 \mathrm{~h}$ nas temperaturas de 23,40 e $60{ }^{\circ} \mathrm{C}$, para as idades de 24 horas, 3, 7 e 28 dias. Nota-se que a temperatura de cura teve forte influência para o CAT/A, onde observa-se que as temperaturas maiores, 40 e $60{ }^{\circ} \mathrm{C}$, propiciaram resistências mais elevadas nas idades iniciais, mas aos 28 dias, os valores mais altos foram à $23 \mathrm{e}$ $40{ }^{\circ} \mathrm{C}$. A temperatura de $40{ }^{\circ} \mathrm{C}$ mostrou-se eficiente para elevar a resistência inicial e tardia (28 dias). Já', para o CAT/B, a cura térmica teve um efeito muito pequeno, já que as resistências se apresentaram baixas para todas as temperaturas e em todas as idades.
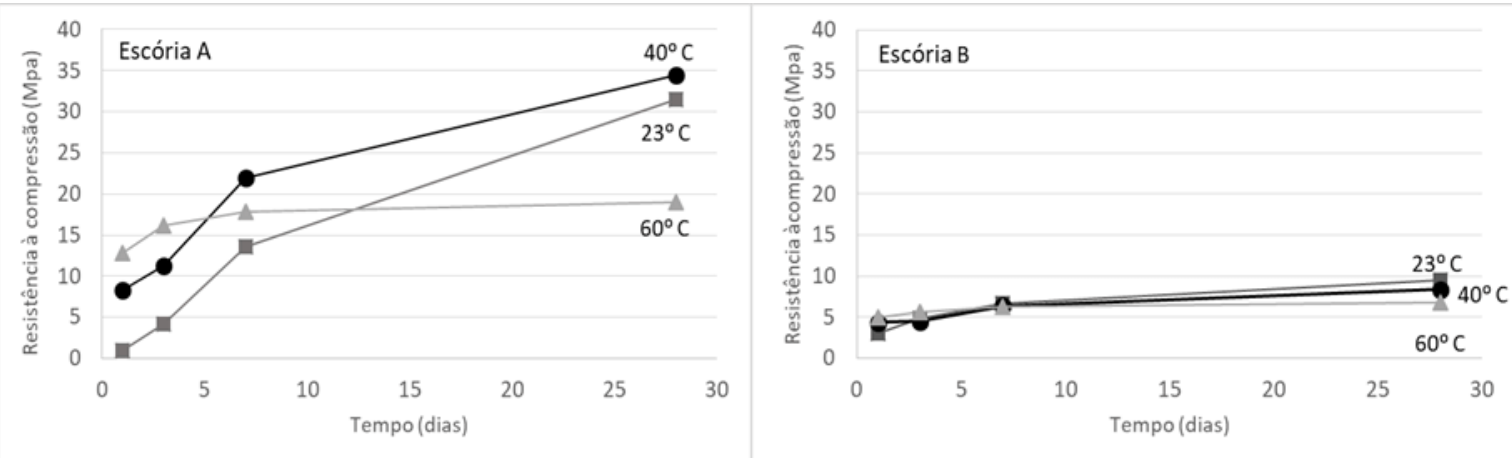

Figura 3: Resistência à compressão dos CAT’s com escória A (CAT/A) e B (CAT/B) sujeitos à cura térmica.

A Figura 4 representa os difratogramas dos CAT's feito com escória A e B, aos7 e 28 dias. Nota-se que em ambos os cimentos, foi identificado o pico de CSH em torno de $29^{\circ}(2 \theta)$, conforme também observado nos trabalhos de Ben Haha et al. e Suwan et al. [10, 11, 12, 19]. Além disso, para o CAT/B, também foi identificada a hidrotalcita em $11^{\circ}$ e $23^{\circ}$, em todas as idades e temperaturas. Para o CAT/A, observou-se que o efeito da temperatura mais alta $\left(60^{\circ} \mathrm{C}\right)$ inibiu a formação do $\mathrm{CSH}$ cristalino. Para o CAT/B, percebeu-se que a cura térmica teve pouco efeito na intensidade do pico de $\mathrm{CSH}$, aos 7 ou aos 28 dias.

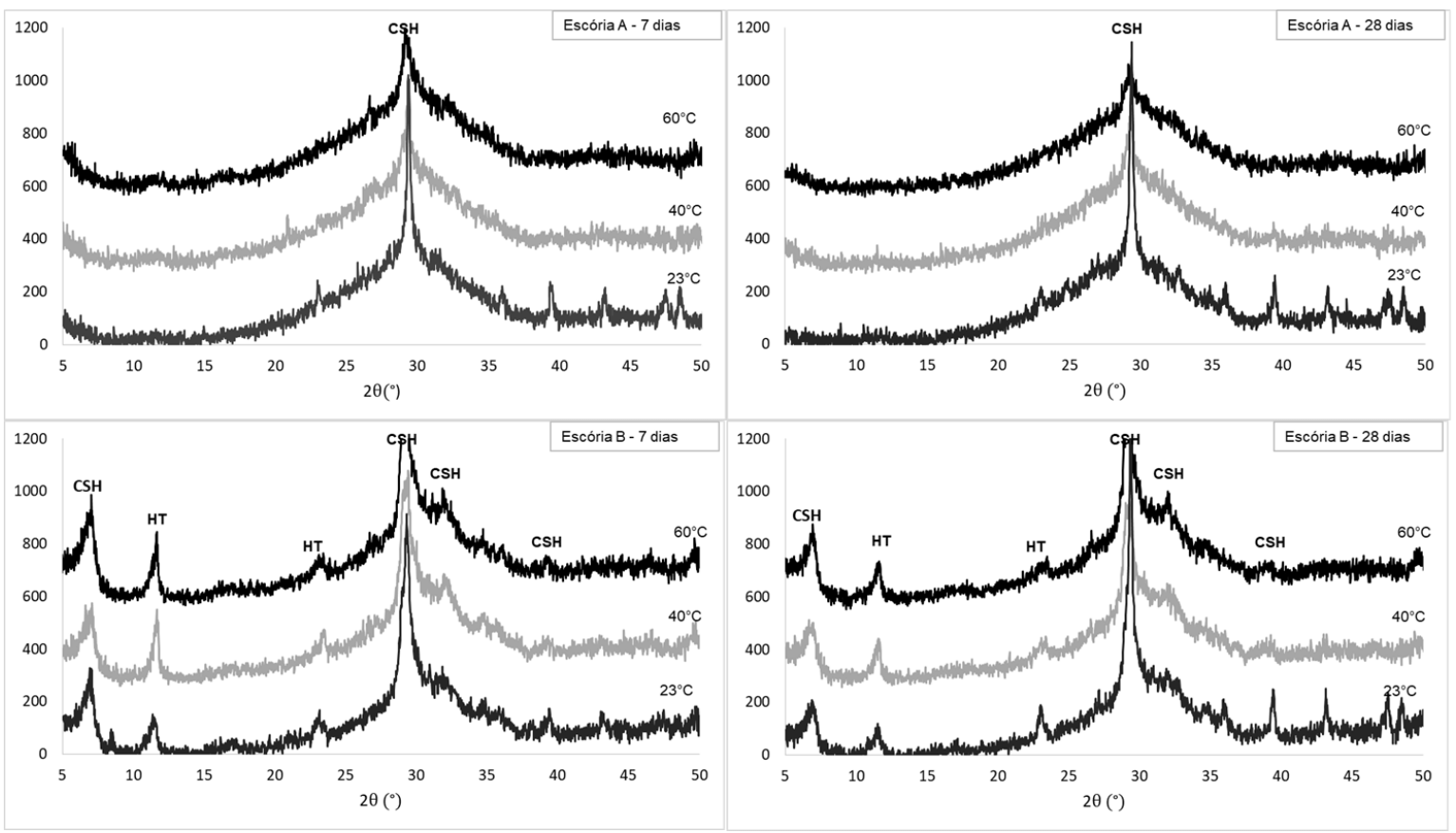


Figura 4: Difratograma dos CAT's com escória A (CAT/A) e B (CAT/B) sujeitos à cura térmica. CSH - Silicato de cálcio hidratado, HT - Hidrotalcita.

A Figura 5 apresenta as análises térmicas (DSC), onde também foi possível observar a presença de compostos hidratados identificados nos difratogramas (CSH e hidrotalcita) e no trabalho de Langaro et al [2]. Para o CAT/A, as curvas de DSC confirmam o efeito benéfico das temperaturas mais altas na formação do CSH aos 7 dias, mas que se mantém similar aos 28 dias. Já, para o CAT/B, a DCS confirma a pequena influência da temperatura de cura no comportamento do cimento, conforme também constado nos testes de resistência à compressão e análise de DRX.

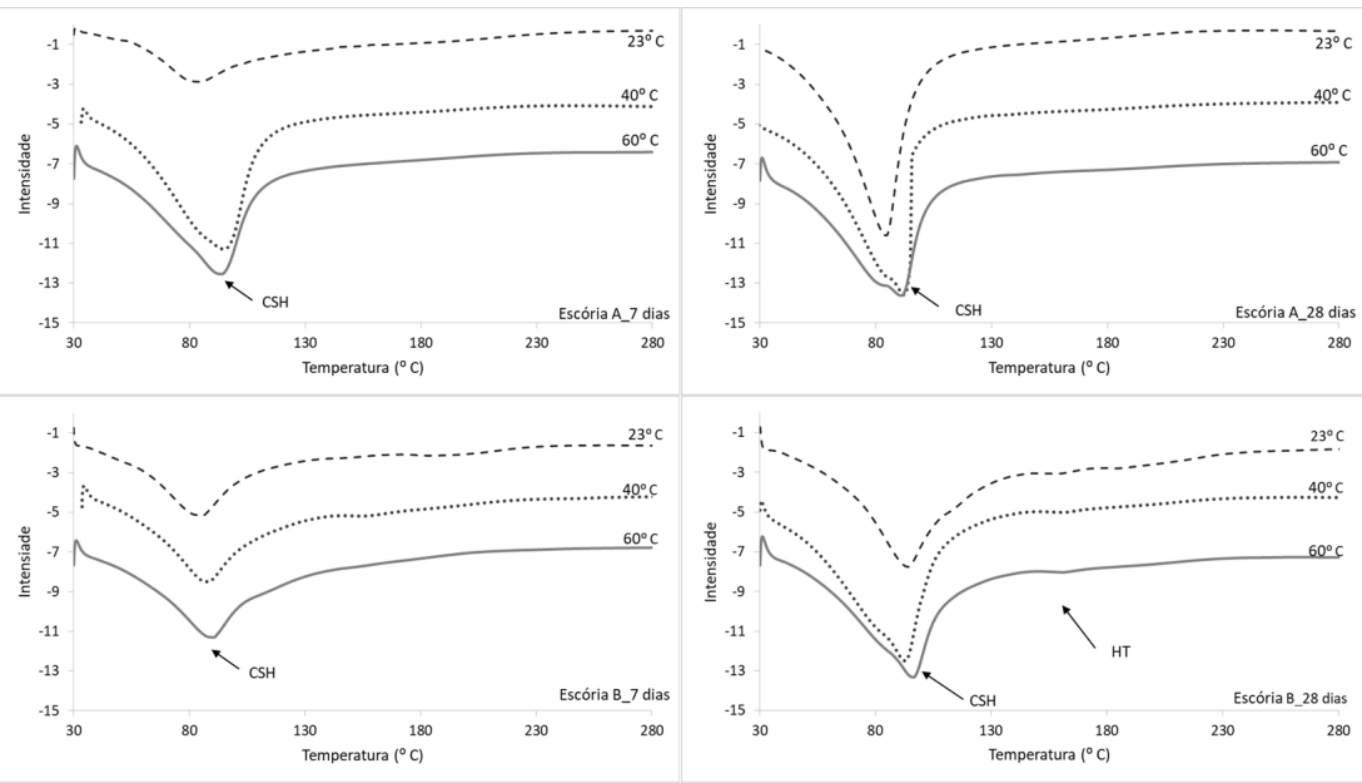

Figura 5: Calorimetria exploratória diferencial dos CAT's com escória A (CAT/A) e B (CAT/B) sujeitos à cura térmica CSH - Silicato de cálcio hidratado, HT - Hidrotalcita.

Com o intuito de observar os compostos hidratados da CAT, realizou-se a microscopia eletrônica de varredura (MEV), cujas imagens estão apresentadas nas Figuras 6 e 7, onde também é possível notar a evolução da microestrutura da pasta com o efeito da temperatura.

Nota-se que o principal composto hidratado (CSH) está presente em todas as amostras. No CAT/A, pode-se observar a presença de fissura aos 7 dias, à $40^{\circ} \mathrm{C}$, a qual pode estar relacionada à intensa retração, que é típica deste cimento e que pode ter sido intensificada pelo efeito da cura térmica. Percebe-se também que à $40{ }^{\circ} \mathrm{C}$ o CSH se apresentou na forma de gel, enquanto que à $60{ }^{\circ} \mathrm{C}$ na forma mais reticulada, o que pode ter favorecido o ganho de resistência nesta temperatura ( $40^{\circ} \mathrm{C}$ ) [26]. Além disso, a estrutura da pasta apresenta-se mais compacta à 23 e $40{ }^{\circ} \mathrm{C}$.
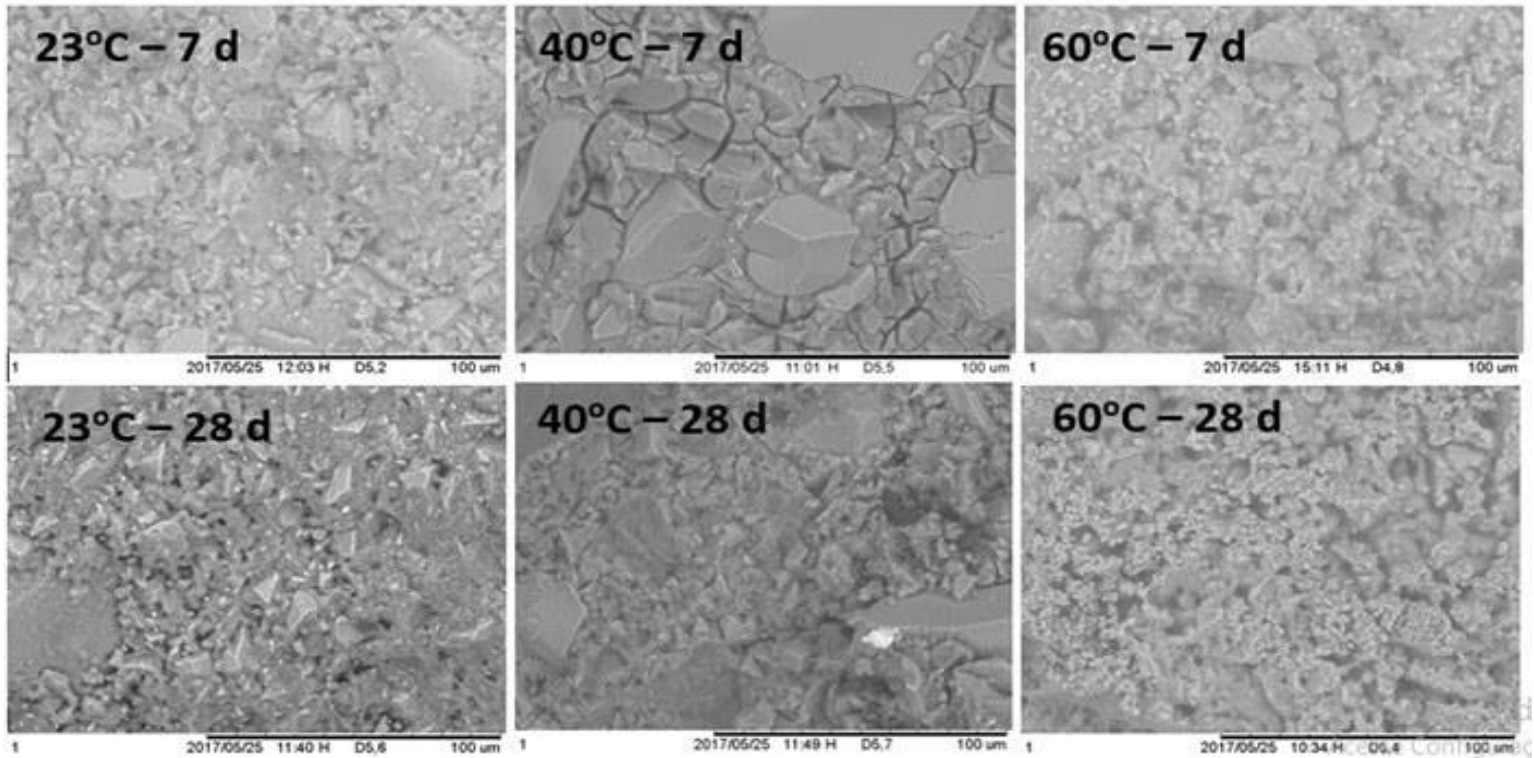
Figura 6: MEV dos CAT's com escória A (CAT/A) sujeitos à cura térmica aos 7 e 28 dias.

Já para a escória B, Figura 7, o CSH apresenta-se mais reticulado, em todas as idades e temperaturas de cura. Também é possível confirmar a presença de hidrotalcita (HT), observada nas DRX's e DSC's, principalmente aos 7 dias. As imagens também confirmam a pouca influência da temperatura de cura na morfologia do CAT/B, além de uma estrutura menos compacta em relação àquela do CAT/A.
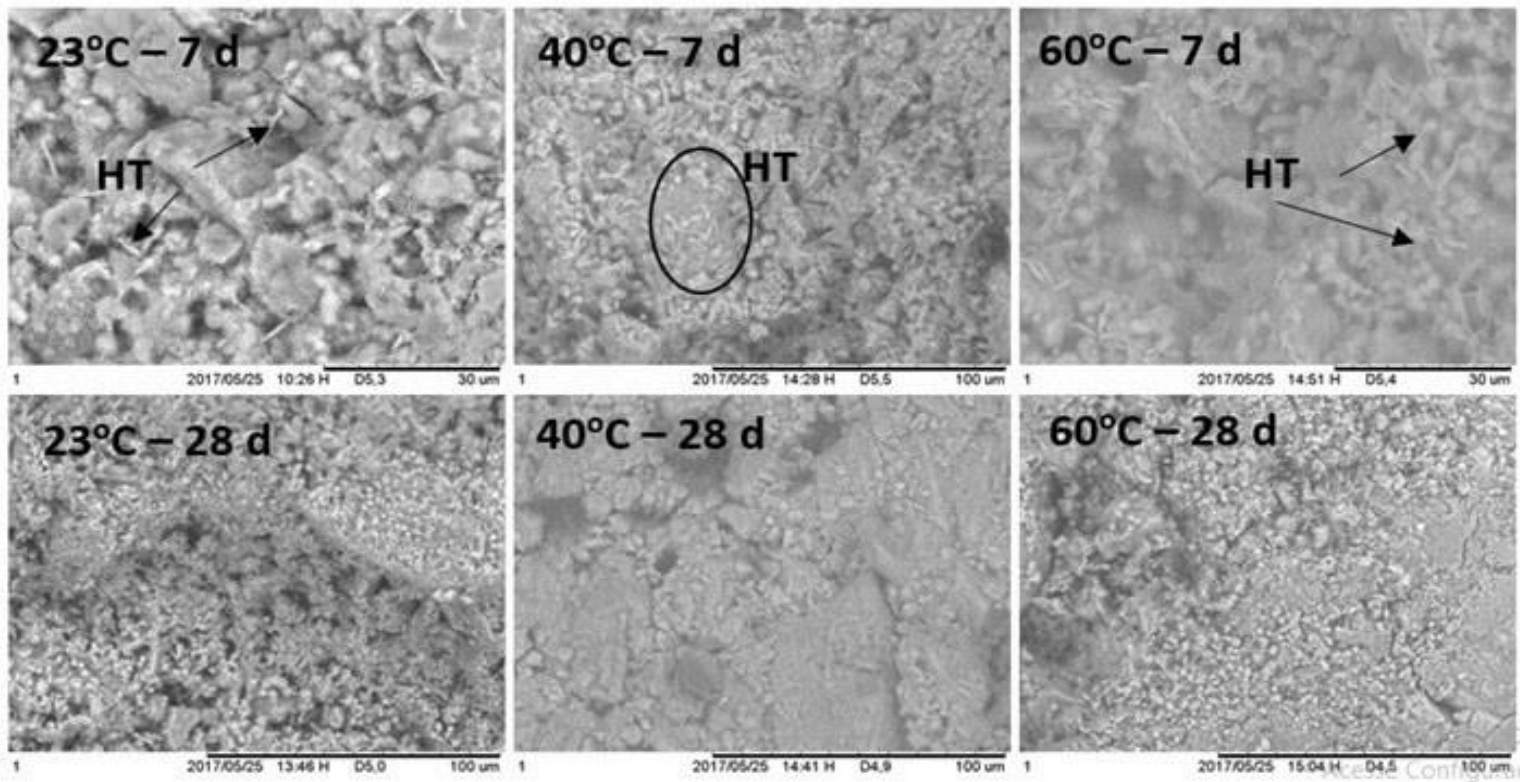

Figura 7: MEV dos CAT's com escória B (CAT/B) sujeitos à cura térmica aos 7 e 28 dias. .

\section{DISCUSSÃO}

Neste trabalho, observou-se que a composição química da escória apresenta grande influência no desenvolvimento de resistência mecânica do cimento álcali-ativado. Os valores apresentados pelas argamassas produzidas com a escória $\mathrm{A}$, são consideravelmente maiores que os apresentados pela escória $\mathrm{B}$. Isto indica que o teor de $\mathrm{MgO}$ pode ter contribuído para o desenvolvimento de resistência mecânica [2, 10, 12], o qual é superior para a escória A.

Em relação à cura térmica, notou-se que para a escória $\mathrm{A}$, a influência da temperatura foi bastante significativa. Nas idades iniciais, os melhores resultados foram obtidos à $60{ }^{\circ} \mathrm{C}$, porém, sem acréscimo de resistência até os 28 dias. A cura à $40{ }^{\circ} \mathrm{C}$ se mostrou eficiente para aumentar a resistência inicial e alcançar $35 \mathrm{MPa}$ aos 28 dias, cujo valor foi superior às demais temperaturas. Para a escória $\mathrm{B}$, o regime de cura não foi capaz de aumentar a resistência mecânica, pois valores foram inferiores a $10 \mathrm{MPa}$.

Através das análises de microestrutura, identificou-se a formação do CSH, para a escória A, e CSH e hidrotalcita para escória B. As análises de DSC confirmaram que, para o CAT/A, o aumento na formação de $\mathrm{CSH}$ em idades iniciais foi fomentado por temperaturas mais altas, o que provavelmente favoreceu o amento da resistência inicial. Todavia, para o CAT/B, constatou-se a incapacidade de aumento de CSH através da temperatura e, consequentemente, não houve melhora no comportamento mecânico.

A formação do CSH a partir da hidratação de escórias é geralmente rico em alumínio, sendo que maior quantidade $\mathrm{Al}_{2} \mathrm{O}_{3}$ pode aumentar a quantidade de $\mathrm{Al}$ no $\mathrm{CSH}$, tornando -se mais amorfo, o que pode explicar o ocorrido com as pastas de escória A [2,11, 24]. Ainda, segundo Taylor [26], as composições com maior conteúdo amorfo nas análises de DRX, apresentam melhores resistências, o mesmo foi observado nas pastas de escória A, que apresentaram maior resistência à compressão e estrutura menos cristalina.

\section{CONCLUSÕES}

Neste estudo apresentou-se a análise da temperatura de cura de cimentos álcali ativados de composição química distintas, visando à obtenção de melhores resultados de resistência à compressão. Através dos resultados, é possível fazer as seguintes considerações:

A escória básica utilizada na produção de CAT não alcançou bons resultados de resistência à com- 
pressão, nem quando submetida à cura térmica. Já, o CAT produzido com escória ácida, alcançou resistência mecânica mesmo à $23^{\circ} \mathrm{C}$, além de ter tido a resistência inicial aumentada pelo efeito da temperatura.

Considerando as temperaturas de cura, os melhores resultados se deram aos $40^{\circ} \mathrm{C}$, onde a resistência inicial se fez superior à da cura em temperatura ambiente, e manteve-se com melhores resultados aos 28dias.

Em relação à microestrutura, o C-S-H foi observado em ambas as escórias, enquanto a hidrotalcita apenas na escória $\mathrm{B}$. $\mathrm{O}$ efeito da temperatura foi similar àquele observado na resistência à compressão, onde a cura teve influência apenas na escória A e pouco (ou nulo) na escória B. O aumento da resistência inicial da resistência para a escória $\mathrm{A}$ foi atribuído à maior formação de CSH.

A morfologia do CSH formado no CAT/A se apresentou mais amorfa, em todas as temperaturas, o que provavelmente influenciou positivamente o desenvolvimento da resistência mecânica.

\section{BIBLIOGRAFIA}

[1] TOPÇU, I.B., TOPRAK, M.U., UYGUNOGLU, T. "Durability and microstructure characteristics of alkali activated coal bottom ash geopolymer cement", J. Clean. Prod., v.81, pp. 211e217, 2014.

[2] LANGARO, E. A., ANGULSKI DA LUZ, C., BUTH, I. S., et al., "The influence of chemical composition and fineness on the performance of alkali activated cements obtained from blast furnace slags", Revista Matéria, v.22, n.1, 2017.

[3] AITCIN, P. C., MINDESS, S. Sustainability of Concrete, 1 ed. USA e Canada, Spon Press, 2011.

[4] DUXSON, P., PROVIS, J. L., LUKEY, G. C., et al., "The role of inorganic polymer technology in the development of "green concrete", Cement and Concrete Research, v. 37, pp. 1590-1597 ., 2007.

[5] MALARD, A. A. M. Avaliação ambiental do setor de siderurgia não integrada a carvão vegetal do estado de Minas Gerais. 2009. 216f. Dissertação de M.Sc., Programa de Pós-Graduação em Sustentabilidade Socioeconômica e Ambiental. Universidade Federal de Ouro Preto, Ouro Preto, Minas Gerais, 2009.

[6] MASSUCATO, C. J. "Utilização de escória de alto-forno à carvão vegetal como adição no concreto", Dissertação de M.Sc., Programa de Pós-Graduação da Universidade Estadual de Campinas, Faculdade de Engenharia Civil, Arquitetura e Urbanismo. Campinas, São Paulo, 2005.

[7] JOHN, M. V., "Cimentos de escória ativada com silicatos de sódio", Tese de D.Sc., Programa de PósGraduação em Engenharia de Construção Civil da Escola Politécnica da Universidade de São Paulo. São Paulo, 1995.

[8] PAL, S. C, MUKHERJEE, A., PATHAK, S. R. "Investigation of hydraulic activity of ground granulated blast furnace slag in concrete", Cement and Concrete Research, v. 33, ed. 9, pp. 1481-1486, 2003.

[9] ALONSO, S., PALOMO, A. "Alkaline activation of metakaolin and calcium hydroxide mixtures: influence of temperature, activator concentration and solids ratio", Materials Letters, v. 47, pp. 55-62, 2001.

[10] BEN HAHA, M., LE SAOUT, G., WINNEFELD, F., et al., "Influence of activator type on hydration kinetics, hydrate assemblage and microstructural development of alkali activated blast-furnace slags", $\mathrm{Ce}$ ment and Concrete Research, v. 41, ed. 3, pp. 301-310 ., 2011.

[11] BEN HAHA, M., LOTHENBACH, B., LE SAOUT, G., et al., "Influence of slag chemistry on the hydra-tion of alkali-activated blast-furnace slag - Part II: Effect of Al2O3", Cement and Concrete Research, v. 42, ed. 1, pp. 74-83., 2012.

[12] BEN HAHA, M., LOTHENBACH, B., LE SAOUT, G., et al., "Influence of slag chemistry on the hydration of alkali-activated blast-furnace slag - part I: effect of MgO", Cement and Concrete Research, v. 41, ed. 9, pp. 955-963 ., 2011.

[13] SCHNEIDER, J., CINCOTTO, M. A., PANEPUCCI, H. "29Si and 27Al high resolution NMR characterization of calcium silicate hydrate phases in activated blast-furnace slag pastes", Cement and Concrete Research, v. 31, ed. 7, pp. 993-1001, 2001.

[14] CHINDAPRASIRT, P., CHAREERAT, T., SIRIVIVATNANON, V. "Workability and strength of coarse high calcium fly ash geopolymer", Cem. Concr. Compos., v.29, pp. 224e229, 2007.

[15] NURUDDIN, M.F., KUSBIANTORO, A., QAZI, S., et al., "Compressive strength and interfacial transition zone characteristic of geopolymer concrete with different cast in-situ curing condition", World Acad. Sceience, Eng. Technol. (WASET), pp.25-28, 2011.

[16] DEEVASAN, K.K.; RANGANATH, R.V., "Geopolymer concrete using industrial byproducts”, Constr. Mater, v.164, (2010). 
[17] CHINDAPRASIRT, P., CHAREERAT, T., HATANAKA, S., et al., "High-strength geopolymer using fine high-calcium fly ash", J. Mater. Civ. Eng., v. 23, n.3, pp. 264-270, 2010.

[18] ALTAN, E., ERDOGAN, S. T., "Alkali activation of a slag at ambient and elevated temperatures", $\mathrm{Ce}$ ment \& Concrete Composites, v. 34, pp. 131-139 ., 2012.

[19] SUAWAN, T., et al., "Micro-mechanisms and compressive strength of Geopolymer-Portland cementitious system under various curing temperatures", Materials Chemistry and Physics, v.180, pp. 219-225, 2016.

[20] FERNÁNDEZ-JIMÉNEZ, A., PALOMO, J., PUERTAS, F., “Alkali activated slag mortars. Mechanical strength behavior", Cement and Concrete Research, v. 29, pp. 1313-1321, 1999.

[21] NURUDDIN, M.F., KUSBIANTORO, A., QAZ, S.A., et al., "Utilisation of Waste Material in Geopolymeric Concrete", Construction Materials, v. 164, Issue CM6, ICE Publishing, pp. 315-327, 2011.

[22] MCNAUGHT, A.D. Compendium of Chemical Terminology, Blackwell Science Oxford, 1997.

[23] ASSOCIAÇÃO BRASILEIRA DE NORMAS TÉCNICAS. NBR 16372. Cimento Portland e outros materiais em pó - Determinação da finura pelo método de permeabilidade ao ar (método de Blaine), Rio de Janeiro, 2015.

[24] AMERICAN SOCIETY FOR TESTING AND MATERIALS. ASTM C348/2: Standard Test Method for Flexural Strength of Hydraulic-Cement Mortars. Estados Unidos, 2002.

[25] CHEN, W., BROUWERS, H. J. H. "The hydration of slag, part 1: reaction models for alkali-activated slag”, Journal of Materials Science, v.42, ed. 2, pp. 428-443, 2007.

[26] TAYLOR, H. F. W. Cement Chemestry, 2 ed, Thomas Telford, 1997.

ORCID

Andreza Frare

Caroline Angulski da Luz https://orcid.org/0000-0003-2395-2947

https://orcid.org/0000-0002-8007-1620 